http://jmscr.igmpublication.org/home/ ISSN (e)-2347-176x ISSN (p) 2455-0450

crossref DOI: https://dx.doi.org/10.18535/jmscr/v8i4.50

\title{
Treatment of Ureteral Stones in Pregnant Women: Role of Semi-Rigid Ureteroscopy and Laser Lithotripsy in the Tertiary Care Center
}

\author{
Authors \\ Dr Deepak Kumar ${ }^{1}$, Dr Namita Gupta ${ }^{2 *}$ \\ ${ }^{1}$ Classified Specialist, Surgical\& Urologist, Command Hospital, Southern Command, Pune \\ ${ }^{2}$ Associate Consultant (Obstetrician \& Gyanecologists), Ruby Hall clinic, Wanowarie, Pune \\ *Corresponding Author \\ Dr Namita Gupta
}

\begin{abstract}
Background: To present our experience with semi-rigid ureteroscopy (URS) and laser lithotripsy in the treatment of ureteral stones in symptomatic pregnant women.

Methods: This study was performed on 55 pregnant women with symptomatic obstructing unilateral ureteral calculi requiring surgical intervention. The patients were treated by URS stone extraction with laser lithotripsy, and Double $J(D J)$ stent placement were routinely done in all patients. The incidence of premature uterine contractions (PUC) due to URS was recorded. In addition to the details of the procedures, serum magnesium, calcium and glucose levels were also measured.

Results: All the patients underwent a successful URS with intra-corporeal laser lithotripsy. No perioperative fetal complications were detected, and all patients completed the full term of pregnancy. Seven patients had a postoperative PUC. An increased risk of PUC was found in patients with fever and renal colic at the initial presentation. PUC was more frequent in patients with lower serum magnesium levels. Univariate analysis showed that fever at initial presentation, high serum glucose and lower serum magnesium levels were indicative for PUC. Also, the combination of preoperative complicated renal colic with fever is also a prognostic indicator for development of PUC. Multivariate analysis showed that fever at initial presentation and lower serum magnesium levels are an independent prognostic marker for PUC.

Conclusions: URS with pneumatic lithotripsy is an effective and safe treatment for pregnant women with obstructing unilateral ureteric calculi. Patients with fever at initial presentation and lower serum magnesium level are the most important factors affecting the preterm complications.

Keywords: Ureteral; Stones; Pregnancy; Ureteroscopy; Lithotripsy.
\end{abstract}

\section{Introduction}

The incidence of urinary calculi during pregnancy ranges from 1 in 200 to 1 in 1,500 and may be associated with $40 \%$ of premature births. Urolithiasis in pregnancy is the most common cause of hospitalization in pregnant women due to non-obstetric causes ${ }^{[1]}$.
A conservative approach is often the most appropriate initial treatment for acute renal colic in the pregnant patient ${ }^{[2,3]}$. However, conservative temporizing approaches with ureteric stenting/ percutaneous nephrostomy insertion are other clinical options to relieve the obstruction until the end of pregnancy ${ }^{[4,5]}$. Over the last decade, 
ureteroscopy (URS) has also become a reasonable alternative and one of the most common treatment options for ureteral stones during pregnancy. There have been more studies reporting on URS in pregnancy, demonstrating its safety and efficacy $^{[6-9]}$. Several criteria have been identified as predictive of future potential risks for both mother and fetus ${ }^{[9-11]}$.

However, to our knowledge few authors have reported on the outcome of using semi-rigid URS with pneumatic lithotripsy in the management of obstructing ureteric calculi in these pregnant patients and to what extent such procedure affects the overall clinical course for both the fetus and mother $^{[12-13]}$.

In the present study, we have evaluated the possible predicting factors responsible for the outcome of pregnant women with obstructing calculi managed with semi-rigid URS with pneumatic lithotripsy, with special reference to premature uterine contraction (PUC) and in addition, assessment the safety and effectiveness of such a procedure.

\section{Patients and Methods}

\section{Patients}

Fifty-five pregnant women were diagnosed with unilateral calcular ureteric obstruction. Diagnosis of ureteral obstruction was made based on actual clinical manifestation of the patients, their abdominal ultrasound (US) findings, and detection of hematuria in urinalysis ${ }^{[7,14]}$. Preoperative data of patients age, stage of pregnancy, diagnostic methods, past history of urolithiasis or urological interventions, localization and size of stone and presenting symptoms and signs were reported. Physical examination, routine urinalysis, urine culture, renal function, blood tests, blood culture if necessary, color Doppler US were done to identify the presence of ureteric stone, and hydroureteronephrosis. The duration of renal colic from onset to surgery and duration of the operation were also recorded. Patients with signs of urinary tract infection (UTI) are considered to have a fever at the time of diagnosis, as they have positive urine culture ${ }^{[9]}$. A consultation with the Department of Obstetrics and Gynecology was done for assessment of both maternal and the fetal condition, specifically PUC. PUC is defined as regular contractions of the uterus resulting in changes in the cervix that start before 37 weeks of pregnancy. Changes in the cervix include effacement (the cervix thins out) and dilation (the cervix opens so that the fetus can enter the birth canal $)^{[15]}$. Also, the serum levels of magnesium, calcium and glucose were assayed in blood samples drawn.

All patients had received initial medical conservative treatment. After failure of medical therapy or there is fever, persistent renal colic and progressive hydronephrosis, those patients were assigned to be treated definitively by ureteroscopic stone extraction. Patients with no stones and those having bilateral ureteric obstruction, single kidney, or transplanted kidney were excluded. This study was approved by our local ethics committee and was conducted in accordance with the Declaration of Helsinki, and all the participants provided a written informed consent.

\section{The URS Procedure}

Under strict maternal and fetal care, spinal anesthesia was performed in 51 of patients, while general anesthesia was administered in four patients. Abdominal US was used for obstetric and renal monitoring throughout all procedures. All the URS procedures were performed by the same surgical team. Endoscopy was carried out using a semi-rigid 9.5 F ureteroscopy (Karl Storz, Tuttlingen, Germany) with a safety sliding guide wire $(0.035 \mathrm{~mm})$ inserted into the affected collecting system under US control or with guidance of a ureteral catheter (4 - 6 F). Balloon dilatation of the stenotic ureteric orifice was necessary only in five $(9 \%)$ of these patients. The stones of all patients were fragmented using a Swiss pneumatic lithoclast $2^{\circledR}$ with the aid of the stone cone to prevent proximal stone migration (retropulsion) during ureteric pneumatic lithotripsy. 
Postoperatively, all patients were followed up during pregnancy by obstetric examination aiming to ensure maternal and fetal well-being as well as outpatient urological follow-up represented by clinical assessment, US examination, urinalysis every 4 weeks, and urine culture.

\section{Evaluation of URS procedure}

The evaluation of the URS procedure included the achievement of a stone-free rate (SFR), resolution of hydronephrosis, and a safely completed pregnancy. SFR was defined as absent of stone fragments. Also, the outcomes included postoperative complications like fever, hematuria, hematoma in bladder, mucosal injury, DJ stent migration, stone migration, and urosepsis. Postoperative infection was defined in patients with fever $\geq 38^{\circ} \mathrm{C}$ with pyuria within 1 week after the operation ${ }^{[9]}$.

\section{Statistical Analysis}

The data were analyzed with the statistical package for social sciences, version 20, for windows software (SPSS Inc, Chicago, Illinois). Results are expressed as mean \pm SEM. The Chisquare test was used to compare categorical variables. For analysis of survival data, KaplanMeier curves were constructed and the logrank test for trend was performed. Multivariate analysis was performed using Cox's proportional hazards model with $\mathrm{P}<0.05$ considered statistically significant.

\section{Results}

A total of 55 pregnant patients with unilateral ureteric calculi underwent a definitive URS. The mean age of the patients and the mean gestational age were $26.9(18$ - 39) years and 21.5 (11 - 32) weeks, respectively. Two patients were admitted during the first trimester, 44 in the second and nine in the third trimester of pregnancy. Patients in the first, second and third trimester had a gestational age of $11.5 \pm 0.5,21.8 \pm 0.7$ and 30.9 \pm 0.5 weeks, respectively. The presenting symptoms were renal colic in 36 patients, hematuria in 17 , and irritative voiding symptoms such as urinary frequency, urgency and dysuria in
19 patients. Eleven patients with fever at initial presentation were at greater risk for the presence of PUC when compared to patients without signs of UTI $(\mathrm{P}<0.005)$ (Table 1). Seven patients had past history of stone disease. Abdominal US revealed normal pyelocaliceal system in five cases, right side hydronephrosis in 31 patients and left side hydronephrosis in 19 patients. A total of 41 of those patients had a ureteral stone diagnosed with color Doppler US and the remaining 14 patients during diagnostic URS. The mean stone size determined by pre-operative color Doppler US in 41 patients was $9.6 \mathrm{~mm}(7-13 \mathrm{~mm})$. Thirty-five patients had right and the other $20 \mathrm{had}$ left ureteral stones. Six had mid-ureteric stones and the remaining 49 had stones in the lower ureter (Table 1).

Table 1 Patients Demographic Data of 55 Pregnant women

\begin{tabular}{|l|c|}
\hline Values & Results \\
\hline Mean age & $26.9(18-39$ years $)$ \\
\hline Mean Follow up & $42.3(6-84$ months) \\
\hline Mean of gestational age (weeks) & $21.5(11-32$ weeks) \\
First trimester (number of patients) & 2 \\
Second trimester (number of patients) & 44 \\
Third trimester (number of patients) & 9 \\
\hline Symptoms & 36 \\
Renal colic & 11 \\
Fever & 17 \\
Hematuria & 19 \\
Irritative symptoms & 50 \\
\hline Hydronephrosis & $53.7 \pm 3.9(12-92) \mathrm{h}$ \\
\hline Time of Renal colic duration & $9.9 \pm 0.7(0.7-2.6) \mathrm{mg} / \mathrm{dL}$ \\
\hline Mean serum Magnesium & $84.8 \pm 3.2(70-160) \mathrm{mg} / \mathrm{dL}$ \\
\hline Mean serum Calcium & \\
\hline Mean serum Glucose & \\
\hline
\end{tabular}

Stone fragments of 50 patients were left to spontaneous passage $(<3 \mathrm{~mm})$ while those of other five patients were extracted using forceps. The stone fragments retrograde migrated to the renal pelvis in five patients. In all patients $6 \mathrm{~F} / 26$ $\mathrm{cm}$ DJ stents were routinely placed. In 50 cases, DJ stents were extracted 2 - 4 weeks after the initial procedure. In five patients with both ureteric dilatation and migrated residual stones, the stent was extracted after successful pregnancy completion. In those five patients, we changed the stent every 6 weeks to restrain encrustation. Minor intraoperative complications such as ureteral edema, mild ureteral laceration or bleeding were encountered in six patients. 
The mean time elapsed until operation and duration of the operation were $66.1 \pm 2.9 \mathrm{~h}$ and $32.8 \pm 0.8 \mathrm{~min}$, respectively. The mean lithotripsy time duration was $16.2 \pm 0.5 \mathrm{~min}$. Also, the mean hospitalization period was $97.2 \pm 6.5 \mathrm{~h}$. Of the 55 patients with ureteral calculi, 50 patients had complete fragmentation of calculi. SFR were $91 \%$. No serious peri-operative, intraoperative urological complications or intraoperative fetal and obstetric complications were observed. All pregnancies were carried to full term and their babies were born normally. The hydronephrosis was relieved in all patients. PUC was observed after the procedure in seven patients who were successfully treated by tocolytics. There was a postoperative fever in six patients who were treated by a safe group of antibiotics. Persistent irritative voiding symptoms were reported by 12 patients. Postoperatively, renal colic developed in three patients who were treated with acetaminophen and prolonged hematuria in six cases (Table 2).

Table 2 Details of Procedure and Outcome of 55 Pregnant Women

\begin{tabular}{|l|c|}
\hline Values & Outcome \\
\hline $\begin{array}{l}\text { Stone location } \\
\text { Lower Ureter }\end{array}$ & $49(89 \%)$ \\
\hline Middle Ureter & $6(11 \%)$ \\
\hline $\begin{array}{l}\text { Stone Laterality (Side of complaint) } \\
\text { Left }\end{array}$ & $20(36 \%)$ \\
\hline Right & $35(64 \%)$ \\
\hline History of Urolithiasis & $7(13 \%)$ \\
\hline Stone diagnosed by Ultrasound & $41(75 \%)$ \\
\hline Mean Stone Size on Ultrasound & $9.6(7-13 \mathrm{~mm})$ \\
\hline Stone diagnosed by Ureteroscopy & $14(25 \%)$ \\
\hline Type of anesthesia & $51(93 \%)$ \\
Spinal & $4(7 \%)$ \\
\hline General & $55(100 \%)$ \\
\hline Type of Lithotripter (Holmium:YAG & \\
Lithotripsy) & $32.8(25-42 \mathrm{~min})$ \\
\hline Duration Of the operation(min) & $66.1 \pm 2.9(33-96 \mathrm{hrs})$ \\
\hline Time elapsed until operation & $16.2(13-25 \mathrm{~min})$ \\
\hline Duration of lithotripsy (min) & $5(9 \%)$ \\
\hline Balloon dilation of ureteral orifice & $55(100 \%)$ \\
\hline Double J stent insertion & $50(91 \%)$ \\
\hline Stone free rate(\%) & $7(13 \%)$ \\
\hline Postoperative complications & $6(11 \%)$ \\
Preterm uterine contraction & $3(5 \%)$ \\
Fever & $6(11 \%)$ \\
Colic & $55(100 \%)$ \\
Hematuria & $97.2 \pm 6.5(24-168 \mathrm{Mnths)}$ \\
\hline Irritative symptoms & \\
\hline Hafely completed pregnancy & \\
\hline
\end{tabular}

The mean serum levels of magnesium, calcium and glucose were $1.9 \pm 0.7(0.7-2.6 \mathrm{mg} / \mathrm{dL}), 9.9$ $\pm 0.2(6.5-7.8 \mathrm{mg} / \mathrm{dL})$ and $84.3 \pm 3.2(70-160$ $\mathrm{mg} / \mathrm{dL}$ ), respectively (Table 1 ). Additionally, the serum magnesium levels of patients were significantly lower in patients with PUC than in patients without PUC $(\mathrm{P}<0.001)$. Interestingly, the opposite relationship was detected; patients with PUC had significantly higher levels of serum glucose $(\mathrm{P}<0.005)$. On the other hands, the serum calcium did not have any significant relationship with PUC (P > 0.05) (Table 3).

Table 3 Univariate Analysis of Prognostic Markers

\begin{tabular}{|l|c|c|}
\hline \multirow{2}{*}{ Variable } & \multicolumn{2}{|c|}{ Outcome parameter } \\
\cline { 2 - 3 } & $\mathbf{X}^{\mathbf{2}}$ & $\mathbf{P}^{*}$ \\
\hline Renal colic & 2.2 & 0.15 \\
\hline Fever & 7.9 & 0.005 \\
\hline Combination preoperative colic and fever & 10.8 & 0.001 \\
\hline Duration of lithotripsy time>15 min & 2.1 & 0.15 \\
\hline Serum Magnesium & 9.5 & 0.002 \\
\hline Serum Glucose & 6.1 & 0.01 \\
\hline *Logrank & \multicolumn{2}{|l}{} \\
\hline
\end{tabular}

The renal colic, fever at the time of diagnosis and duration of lithotripsy of more than $15 \mathrm{~min}(\mathrm{P}<$ 0.05 ) have a statistically significant relationship to PUC. However, the following parameters: duration of the operation, time elapsed of more than $36 \mathrm{~h}$ until operation, types of anesthesia, stone localization, stone diameter, SFR at the end of procedure, hospitalization length and postoperative fever and colic did not have a statistically significant relationships in patients with PUC.

Univariate analysis, using the logrank test, showed a prognostic impact for those having fever at the time of diagnosis, serum magnesium, and serum glucose level on PUC. The time of lithotomy operation had no significant influence on the course of PUC. Also, the presence of postoperative fever and renal colic was of no prognostic significance (Table 3).

To test whether any of these parameters had a prognostic impact, a multivariate Cox regression analysis was done including the parameters: fever at the time of diagnosis, serum level of magnesium and serum level of glucose for PUC. The parameters that were not dichotomic were dichotomized and the cut-off of serum magnesium and glucose $(<1.7$ versus $>1.7 \mathrm{mg} / \mathrm{dL},<10.1$ 
versus > $10.1 \mathrm{mg} / \mathrm{dL}$, respectively). In that analysis, fever and serum magnesium could be identified as an independent prognostic marker for poor prognosis.

By combining renal colic and fever at the time of diagnosis, four patient groups were considered (Group 1: patients with positive renal colic and fever at the time of diagnosis, Group 2: those with positive renal colic and without fever, Group 3: those without renal colic and with fever and Group 4: those without renal colic and fever). The Kaplan-Meier curve shows the prognostic influence of those with positive renal colic and fever on the time to progression to PUC. Clearly, patients with renal colic and fever had a statistically significant poor prognosis compared to the other groups (Table 3). Generally, this data are consistent with the notion that patients without renal colic and fever do better than those with positive renal colic and fever. Patient with fever and renal colic were simultaneously present in eight (15\%) patients of whom seven (87\%) developed PUC.

\section{Discussion}

Technological advancements in the development of the semi-rigid ureteroscopes have enabled atraumatic fragmentation of stones in pregnant women $^{[16]}$. It was reported that URS with lithotripsy is an ideal intervention for treatment of pregnant women with obstructing lower ureteric calculi that did not respond to conservative treatment. This method is effective in reducing pain, preventing UTI and decreasing obstetric complications. Many trials were reported on the safety and efficacy of URS in all trimesters in pregnant women. Also, in this recurrent study, all pregnant patients completed the pregnancy to full term with no serious obstetric or fetal complications $^{[4,17-19]}$. However, PUC developed in seven patients in this current study.

Previous studies on the risk factors for developing PUC in pregnant women with ureteric calculi had been linked to renal colic and urosepsis ${ }^{[11]}$. In addition, serum magnesium, glucose levels and
SFR at the end of procedure have been described as potential factors ${ }^{[9]}$. However, there are many possible causes for serious postoperative complications, related to URS procedures. So, it remains difficult to predict accurately from the initial evaluation which patients will have an unfavorable clinical outcome.

In this current study, renal colic has a statistically significant condition with the development of PUC $^{[11,20]}$. Its role during pregnancy in the development of PUC is unknown. However, urolithiasis and episodes of renal colic can lead to the development of hydronephrosis and urinary stasis and eventually UTI, which can result in obstetric complications ${ }^{[21]}$. In pregnant patients with complicated pain, URS can be used as both diagnostic and therapeutic procedure due to a potentially high number of diagnosed ureteral stones, that was reflected by our results, where $25 \%$ of ureteric stone was detected during diagnostic $\mathrm{URS}^{[4,18,22]}$. In pregnant women, dilatation of the ureteric orifices during URS is often unnecessary due to the muscle-relaxing effect of progesterone which elevated during pregnancy ${ }^{[16]}$. However, in the present study a stenotic ureteric orifice was dilated up to $12 \mathrm{~F}$ in five cases, something that was reflected by others studies $^{[13,23]}$.

We successfully fragmented all stones localized in the ureter using laser lithotripter without any serious obstetric complications ${ }^{[12,22]}$. The laser lithoclast used here can be applied only via a semi-rigid URS. These laser lithotriptors can focus their energy on a localized area with minimal energy dispersion without risks and any traumatic complication during pregnancy ${ }^{[24]}$. In contrast to other stone-fragmentation energy sources e.g., electrohydraulic generators and ultrasonic lithotripsy have different risks during pregnancy. For example, ultrasonic lithotripsy should be avoided due to possible hearing damage to the fetus ${ }^{[25,26]}$. With holmium: YAG lithotripsy, the time taken for stones to fragment into removable size is shorter than pneumatic lithotripsy. But the, holmium laser lithotripsy is 
higher costs compared to pneumatic lithotripsy [18].

In our study, we found that the duration of lithotripsy of more than $15 \mathrm{~min}$ is a risk factor for PUC with a statistically significant correlation. However, the time elapsed of more than $36 \mathrm{~h}$ until operation did not show a statistically significant correlation with PUC, as reflected by Buttice et al ${ }^{[9]}$. But early intervention should be suggested in cases of symptomatic pregnant patients, whereas, the delayed operation was found to be a risk factor for urosepsis development ${ }^{[9]}$.

In these cases, DJ stents were routinely inserted in all patients after URS procedures to ensure adequate drainage of the obstructed ureter and reduce the postoperative pain and analgesic use [27]. No significant complication related to its insertion occurred in the current study. However, some risks such as UTI and encrustation have been reported ${ }^{[23,28]}$. This encrustation requires frequent stent changes, which can be every $6-8$ weeks.

In our patients, we correlated PUC to a specific laboratory parameter. Low serum magnesium level has been found to unfavorably correlate with PUC, as about $85 \%$ of patient with low magnesium level developed PUC. PUC probably be due to the loss of inhibitory effect of magnesium on PUC which is mediated through the antagonism of calcium-mediated uterine contractions. But, the high serum glucose level is associated with increased risk of progression to PUC. The significant increase in serum glucose levels in patients with PUC can be attributed to increased catabolic activity in sepsis patient, which is associated with septicemia ${ }^{[29]}$. However, serum calcium level did not preclude any relation to PUC which reflected by Buttice $S$ et al ${ }^{[9]}$.

However, the nature of PUC in pregnant patients with ureteric stone is still debatable and may due to a variety of factors. Stone size, SFR, types of anesthesia, hydronephrosis, in addition, postoperative colic and fever complication are other important factors accounting for PUC. Although such factors did have a statistically significant correlation with PUC, these variable observations suggest that PUC outcome after laser lithotripsy is probably determined by a combined effect of various factors. In the present study, the combination of both preoperative renal colic and fever at initial presentation were associated with the worst long-term prognosis for PUC; as all the patients with this combination developed $\mathrm{PUC}^{[30]}$. The limitations in this study include the relatively low numbers of patients. Also, evaluating all the parameters including blood tests, radiology, timing, proficiency of the surgeon, general status of the fetus and patient could not be carried out to provide exact mean values.

Our study provides evidence that complicated renal colic with fever and low serum magnesium level have an influence on PUC in pregnant patients who have ureteral stone. If these results are confirmed in other studies, both factors could emerge as additional parameters for predicting the clinical outcome of pregnant patients with urolithiasis. Also, the results of this study suggest that for pregnant patients with refractory unilateral symptomatic stones, semi-rigid URS with laser lithotripsy and DJ stent placement are effective and safe options.

\section{References}

1. Srirangam SJ, Hickerton B, Van Cleynenbreugel B. Management of urinary calculi in pregnancy: a review. J Endourol. 2008;22(5):867-875.

2. Parulkar BG, Hopkins TB, Wollin MR, Howard PJ, Jr., Lal A. Renal colic during pregnancy: a case for conservative treatment. J Urol. 1998;159(2):365-368.

3. Kavoussi LR, Jackman SV, Bishoff JT. Re: Renal colic during pregnancy: a case for conservative treatment. $\mathbf{J}$ Urol. 1998;160(3 Pt 1):837-838.

4. Lifshitz DA, Lingeman JE. Ureteroscopy as a first-line intervention for ureteral calculi in pregnancy. J Endourol. 2002; 16(1):19-22. 
5. Semins MJ, Trock BJ, Matlaga BR. The safety of ureteroscopy during pregnancy: a systematic review and meta-analysis. J Urol. 2009;181(1):139-143.

6. Teleb M, Ragab A, Dawod T, Elgalaly H, Elsayed E, Sakr A, Abdelhameed A, et al. Definitive ureteroscopy and intracorporeal lithotripsy in treatment of ureteral calculi during pregnancy. Arab J Urol. 2014;12 (4):299-303.

7. Adanur S, Ziypak T, Bedir F, Yapanoglu T, Aydin HR, Yilmaz M, Aksoy M, et al. Ureteroscopy and holmium laser lithotripsy: is this procedure safe in pregnant women with ureteral stones at different locations? Arch ItalUrolAndrol. 2014;86(2):86-89.

8. Wymer K, Plunkett BA, Park S. Urolithiasis in pregnancy: a costeffectiveness analysis of ureteroscopic management vs ureteral stenting. Am J Obstet Gynecol. 2015;213(5):691 e691698.

9. Buttice S, Lagana AS, Vitale SG, Netsch C, Tanidir Y, Cantiello F, Dragos L, et al. Ureteroscopy in pregnant women with complicated colic pain: Is there any risk of premature labor? Arch ItalUrolAndrol. 2017;89(4):287-292.

10. Mitrovic-Jovanovic A, Dragojevic-Dikic S, Zamurovic M, Nikolic B, Gojnic M, Rakic S, Jovanovic T. Comparison of electrolytic status $(\mathrm{Na}+, \mathrm{K}+, \mathrm{Ca} 2+, \mathrm{Mg} 2+)$ in preterm and term deliveries. ClinExpObstet Gynecol. 2012;39(4):479482.

11. Zhang S, Liu G, Duo Y, Wang J, Li J, Li C. Application of Ureteroscope in Emergency Treatment with Persistent Renal Colic Patients during Pregnancy. PLoS One. 2016;11(1):e0146597.

12. Laing KA, Lam TB, McClinton S, Cohen NP, Traxer O, Somani BK. Outcomes of ureteroscopy for stone disease in pregnancy: results from a systematic review of the literature. Urol Int. 2012;89(4):380-386.

13. Bozkurt Y, Soylemez H, Atar M, Sancaktutar AA, Penbegul N, Hatipoglu NK, Bodakci MN, et al. Effectiveness and safety of ureteroscopy in pregnant women: a comparative study. Urolithiasis. 2013; 41(1):37-42.

14. Abedi AR, Allameh F, Razzaghi MR, Fadavi B, Qashqai H, Najafi S, Ranjbar A, et al. The Efficacy and Safety of Laser Lithotripsy in Pregnancy. J Lasers Med Sci. 2017;8(2):84-87.

15. Sarri G, Davies M, Gholitabar M, Norman JE, Guideline Development G. Preterm labour: summary of NICE guidance. BMJ. 2015;351:h6283.

16. Georgescu D, Multescu R, Geavlete B, Geavlete P, Chiutu L. Ureteroscopy - firstline treatment alternative in ureteral calculi during pregnancy? Chirurgia (Bucur). 2014;109(2):229-232.

17. Ulvik NM, Bakke A, Hoisaeter PA. Ureteroscopy in pregnancy. J Urol. 1995;154(5):1660-1663.

18. Watterson JD, Girvan AR, Beiko DT, Nott L, Wollin TA, Razvi H, Denstedt JD. Ureteroscopy and holmium:YAG laser lithotripsy: an emerging definitive management strategy for symptomatic ureteral calculi in pregnancy. Urology. 2002;60(3):383-387.

19. Lemos GC, El Hayek OR, Apezzato M. Rigid ureteroscopy for diagnosis and treatment of ureteral calculi during pregnancy. Int Braz J Urol. 2002;28 (4):311-315.

20. Biyani CS, Joyce AD. Urolithiasis in pregnancy. II: management. BJU Int. 2002;89(8):819-823.

21. Boridy IC, Maklad N, Sandler CM. Suspected urolithiasis in pregnant women: imaging algorithm and literature review. AJR Am J Roentgenol. 1996;167(4):869875. 
22. Bozkurt Y, Penbegul N, Soylemez H, Atar M, Sancaktutar AA, Yildirim K, Sak ME. The efficacy and safety of ureteroscopy for ureteral calculi in pregnancy: our experience in 32 patients. Urol Res. 2012;40(5):531-535.

23. Rana AM, Aquil S, Khawaja AM. Semirigid ureteroscopy and pneumatic lithotripsy as definitive management of obstructive ureteral calculi during pregnancy. Urology. 2009;73(5):964-967.

24. Zheng W, Denstedt JD. Intracorporeal lithotripsy. Update on technology. UrolClin North Am. 2000;27(2):301-313.

25. Kroovand RL. Stones in pregnancy and in children. J Urol. 1992;148(3 Pt 2):10761078.

26. Ishii H, Aboumarzouk OM, Somani BK. Current status of ureteroscopy for stone disease in pregnancy. Urolithiasis. 2014;42(1):1-7.

27. Akpinar H, Tufek I, Alici B, Kural AR. Ureteroscopy and holmium laser lithotripsy in pregnancy: stents must be used postoperatively. J Endourol. 2006;20(2):107-110.

28. Johnson EB, Krambeck AE, White WM, Hyams E, Beddies J, Marien T, Shah O, et al. Obstetric complications of ureteroscopy during pregnancy. J Urol. 2012;188(1): 151-154.

29. Chuang CC, Shiesh SC, Chi CH, Tu YF, Hor LI, Shieh CC, Chen MF. Serum total antioxidant capacity reflects severity of illness in patients with severe sepsis. Crit Care. 2006;10(1):R36.

30. Abdel-Kader MS, Tamam AA, Elderwy AA, Gad M, El-Gamal MA, Kurkar A, Safwat AS. Management of symptomatic ureteral calculi during pregnancy: Experience of 23 cases. Urol Ann. 2013; 5(4):241-244. 